\title{
The Hardware-in-the-Loop Simulation (HILS) of the Coordinated Control of the Hybrid Energy Storage System in Microgrid
}

\author{
Rui Hou ${ }^{1,2}$, Thai-Thanh Nguyen ${ }^{1}$, Hak-Man Kim ${ }^{1, *}$, Huihui Song ${ }^{2}$ \\ and Yanbin $\mathrm{Qu}^{2, *}$ \\ ${ }^{1}$ Department of Electrical Engineering, Incheon National University, (Songdo- \\ dong)119 Academy-ro, Yeonsu-gu, Incheon, Korea \\ ${ }^{2}$ School of Information and Electrical Engineering, Harbin Institute of \\ Technology at Weihai, No.2 in Wenhua Xi Road, Weihai, China \\ ${ }^{1}$ hmkim@inu.ac.kr, ${ }^{2}$ quyanbin@hit.edu.cn
}

\begin{abstract}
The hybrid energy storage system (HESS) consisted of the battery, and superconducting magnetic energy storage (SMES) is used in microgrid (MG) to smooth the power fluctuation of wind generation $(W G)$ in this study. One second-order low-pass filter (LPF) with a good frequency selectivity is employed to extract the low-frequency power as the power reference of the battery, which can contribute to extending the battery service life. For the purpose of improving the control performance of HESS, the passivitybased $(P B)$ control method, an advanced nonlinear control strategy, is employed in the current loops. The design process of the PB controller for HESS is proposed in detail by the interconnection and damping assignment (IDA) methodology. The proposed coordination control strategy for HESS is verified in a test MG system by the hardwarein-the-loop simulation (HILS). The real-time simulation results demonstrate that HESS can smooth the WG output power well with a satisfactory coordination performance for the battery and SMES.
\end{abstract}

Keywords: Microgrid (MG); renewable power generation (RPG); hybrid energy storage system (HESS); coordinated control; hardware-in-the-loop simulation (HILS)

\section{Introduction}

The microgrid (MG) has been proved as the most effective way to organize and manage the renewable power generations (RPGs). The common RPGs such as the wind generation (WG), Photovoltaic (PV) generation and tidal power generation have the fluctuating and intermittent characteristics. To overcome this problem, the energy storage system (ESS) play an important role in the MG for the energy buffering, which can improve the stability of the power system $[1,2]$.

The energy storage elements can be classified into two groups. One is the energy type which has a large energy density such as batteries and pumped storage. The drawback of these elements is that they have the low power response speed and cannot charge or discharge very frequently. Another type is called the power type with a large power density such as supercapacitor (SC), superconducting magnetic energy storage (SMES), and flywheel energy storage. These storage elements have the quick power response, but the inadequate storage capacity limits their high-power applications. The single type of energy storage element might not satisfy the need of MG applications owing to their respective defects. To complement each type's advantages and improve the overall performance of ESS, two kinds of the storage elements can combine as the hybrid energy storage system (HESS) [3-5]. Up to now, most of the research on the HESS concern the

${ }^{*}$ Corresponding Authors 
hybrid system consisted of the battery energy storage system (BESS) and SC. In fact, SMES which is almost unlimited by the charge-discharge cycle numbers has the more attractive performance than SC such as the larger power and energy densities [6]. Therefore, the HESS consisted of the battery and SMES is considered in this study.

For the purpose of achieving the coordinated operation of HESS, a low-pass filter (LPF) is usually used to separate the power reference for each storage element [7-10]. For the sake of extending the service life, the battery only generates the low-frequency power. The power type storage element is responsible for the high-frequency power. Many researchers have studied this strategy. Nevertheless, they usually use the simple first order LPF which has a poor frequency selectivity. Some intelligent methods are used to separate the power reference such as the artificial neural network (ANN) [11] and wavelet analysis [12]. However, these methods are hard for the practical implementation owing to the complex calculation.

The current control strategy is critical for the HESS performance. Passivity-based (PB) control, a promising nonlinear control strategy, has the advantage of the outstanding transient performance and strong robustness, which can improve the defects of the conventional proportional-integral (PI) control in the nonlinear system like HESS [13]. There are several methods to realize the PB control, and the interconnection and damping assignment (IDA) strategy is usually employed for the sake of simple implementation and good performance [14]. Refs [15, 16] have applied PB method for the power converter control and showed the better control effects than PI method. For the purpose of achieving the outstanding control performance, PB control is implemented in the proposed HESS system in this study.

Hardware-in-the-loop simulation (HILS) system, an advanced real-time simulation device, can provide the convenient approach for the verification of complex system like HESS with the credible results. This paper presents the implementation and HILS system test of the PB controlled HESS consisted of BESS and SMES. The paper structure is as follows: Section 2 illustrates the topology and control scheme of HESS. Section 3 presents the design of PB controller for HESS. Section 4 depicts the system of the HILS test. Section 5 demonstrates the experimental results. Section 6 concludes this study.

\section{The Topology and Control Scheme of HESS}

As is shown in Figure 1, the HESS considered in this paper comprises the BESS and SMES. BESS contains a voltage source converter (VSC). SMES consists of a VSC and a DC chopper and is called VSC-based SMES. LCL filter is used to absorb the switching ripples of the power converters. BESS and SMES are parallel on the AC side, and this topology is very suitable for the high-power applications. The power of BESS and SMES can be regulated independently. By the coordinated control of HESS, the optimal operation of each ESS can be achieved.

The related variables are defined in Figure 1. A general battery model consisted of a controller voltage source $E_{\mathrm{g}}$ and a series internal resistance $R_{\mathrm{b}}$ is adopted, which can reflect the dynamic behavior of the common batteries ${ }^{[17]}$. The constant parameter $R_{\mathrm{b}}$ can be obtained from the instructions of the battery, and $E_{\mathrm{g}}$ can be calculated by the equation provided in ref [17].

The control scheme of HESS is illustrated in Figure 2. In this study, HESS is used to smooth the power fluctuation of the WG which is a representative of the typical RPG. The LPF1 is used to acquire the average output power of WG. The LPF2 is employed to separate the power reference for BESS and SMES. BESS only generates the lowfrequency power to extend the service life. SMES has no limitation to the chargedischarge cycle numbers with a very fast power response. Therefore SMES delivers the high-frequency power in the HESS to make up the shortcomings of BESS. 
These two LPFs play important roles in the performance of HESS and should be designed carefully. Conventional first-order inertial LPF is usually employed in the references for the simplicity. However, the poor frequency selectivity of the LPF may induce the unsatisfactory effects on the WG power smoothing and the battery protecting. With the full consideration of the performance and implementation difficulty, the secondorder Butterworth IIR LPFs are employed in this study. To smooth the WG well, the LPF1 should have a low bandwidth whose cutoff frequency is chosen as $0.1 \mathrm{~Hz}$ in this study. The cutoff frequency of LPF2 is dependent on the endurance of the battery and determined to be $0.5 \mathrm{~Hz}$. These two LPFs can be designed easily by the toolbox of Matlab.

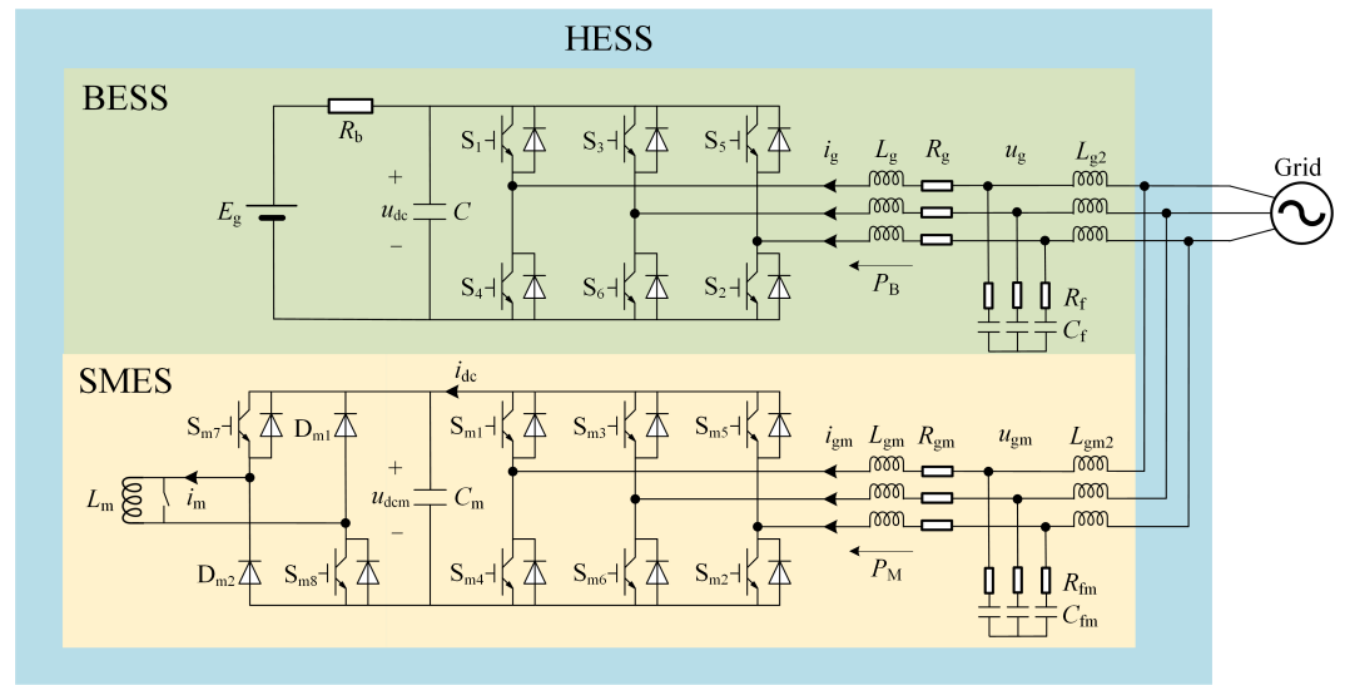

Figure 1. The Topology of HESS

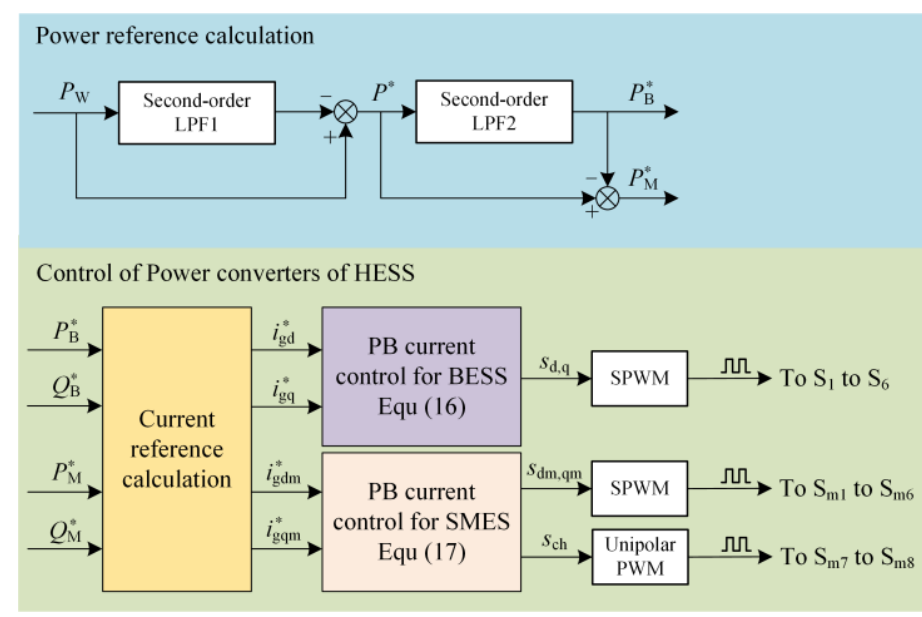

Figure 2. The Control Scheme of HESS

The power reference can directly convert to the current reference of the power converters in the $\mathrm{d}-\mathrm{q}$ frame by the equations as follows: 


$$
\left\{\begin{array}{l}
i_{\mathrm{gd}(\mathrm{m})}^{*}=\frac{2\left(u_{\mathrm{gd}(\mathrm{m})} P_{\mathrm{B}(\mathrm{M})}^{*}+u_{\mathrm{gq}(\mathrm{m})} Q_{\mathrm{B}(\mathrm{M})}^{*}\right)}{3\left(u_{\mathrm{gd}(\mathrm{m})}^{2}+u_{\mathrm{gq}(\mathrm{m})}^{2}\right)} \\
i_{\mathrm{gq}(\mathrm{m})}^{*}=\frac{2\left(u_{\mathrm{gq}(\mathrm{m})} P_{\mathrm{B}(\mathrm{M})}^{*}-u_{\mathrm{gd}(\mathrm{m})} Q_{\mathrm{B}(\mathrm{M})}^{*}\right)}{3\left(u_{\mathrm{gd}(\mathrm{m})}^{2}+u_{\mathrm{gq}(\mathrm{m})}^{2}\right)}
\end{array}\right.
$$

The power regulation adopts the strategy of tracking control without any power feedback due to the consideration of the control system stability. The PB current regulators that will be designed in Section 3 receive the current references as the input and calculate the duty ratio $s$ for the BESS and SMES respectively. Then the sinusoidal pulse width modulation (SPWM) is used to drive the IGBTs in the VSCs of HESS. Unipolar PWM technique serves for the DC chopper to reduce the switching ripple.

\section{The Design of PB Current Controller for HESS}

The mathematical model of the BESS and SMES are constructed firstly and showed in the following equations:

$$
\begin{gathered}
\left\{\begin{array}{c}
L_{\mathrm{g}} d i_{\mathrm{gd}} / d t=-R_{\mathrm{g}} i_{\mathrm{gd}}+\omega L_{\mathrm{g}} i_{\mathrm{gq}}-s_{\mathrm{d}} u_{\mathrm{dc}}+u_{\mathrm{gd}} \\
L_{\mathrm{g}} d i_{\mathrm{gq}} / d t=-R_{\mathrm{g}} i_{\mathrm{gq}}-\omega L_{\mathrm{g}} i_{\mathrm{gd}}-s_{\mathrm{q}} u_{\mathrm{dc}}+u_{\mathrm{gq}} \\
C d u_{\mathrm{dc}} / d t=1.5 s_{\mathrm{d}} i_{\mathrm{gd}}+1.5 s_{\mathrm{q}} i_{\mathrm{gq}}+\left(E_{\mathrm{g}}-u_{\mathrm{dc}}\right) / R_{\mathrm{b}}
\end{array}\right. \\
\left\{\begin{array}{c}
L_{\mathrm{gm}} d i_{\mathrm{gdm}} / d t=-R_{\mathrm{gm}} i_{\mathrm{gdm}}+\omega L_{\mathrm{gm}} i_{\mathrm{gqm}}-s_{\mathrm{dm}} u_{\mathrm{dcm}}+u_{\mathrm{gdm}} \\
L_{\mathrm{gm}} d i_{\mathrm{gqm}} / d t=-R_{\mathrm{gm}} i_{\mathrm{gqm}}-\omega L_{\mathrm{gm}} i_{\mathrm{gdm}}-s_{\mathrm{qm}} u_{\mathrm{dcm}}+u_{\mathrm{gqm}} \\
C_{\mathrm{m}} d u_{\mathrm{dcm}} / d t=i_{\mathrm{dc}}-s_{\mathrm{ch}} i_{\mathrm{m}} \\
L_{\mathrm{m}} d i_{\mathrm{m}} / d t=s_{\mathrm{ch}} u_{\mathrm{dcm}}
\end{array}\right.
\end{gathered}
$$

In the above equations, the VSCs are modeled in the d-q frame to simplify the control. $\omega$ is the fundamental angle frequency of the power grid. Most of the variables have been defined in Figure 1. For the sake of the simplicity the equation, the duty ratio of the DC chopper $s_{\mathrm{ch}}$ is defined as below:

$s_{\mathrm{ch}}=s_{\mathrm{m} 7}+s_{\mathrm{m} 8}-1$

Where $s_{\mathrm{m} 7}$ and $s_{\mathrm{m} 8}$ represent the duty ratio of the IGBTs in the DC chopper. For the purpose of designing the $\mathrm{PB}$ controller, the mathematic model should be converted to the port-controlled Hamiltonian ( $\mathrm{PCH})$ equation whose standard form is shown as below:

$$
\left\{\begin{array}{l}
\dot{x}=(J-R) \nabla H+g u \\
y=g^{\mathrm{T}} \nabla H
\end{array}\right.
$$

Where $u$ and $y$ are the input and output variables of the PCH system. $J$ and $R$ reflect the internal structure characteristics and the dissipation features. $g$ describes the connection structure of the system with outside. $H$ is the Hamiltonian energy function whose value can be determined as the total energy of the storage elements in the system. According to (2) and (3), the related matrices in PCH model of the BESS and SMES are as follows: 


$$
\begin{aligned}
& J_{(\mathrm{b})}=\left[\begin{array}{ccc}
0 & \omega L_{\mathrm{g}} & -s_{\mathrm{d}} \\
-\omega L_{\mathrm{g}} & 0 & -s_{\mathrm{q}} \\
s_{\mathrm{d}} & s_{\mathrm{q}} & 0
\end{array}\right], g_{(\mathrm{b})}=\left[\begin{array}{ccc}
1 & 0 & 0 \\
0 & 1 & 0 \\
0 & 0 & 1
\end{array}\right], R_{(\mathrm{b})}=\left[\begin{array}{ccc}
R_{\mathrm{g}} & 0 & 0 \\
0 & R_{\mathrm{g}} & 0 \\
0 & 0 & \frac{2}{3 R_{\mathrm{b}}}
\end{array}\right] \\
& \left\{\begin{array}{l}
x_{(\mathrm{b})}=\left[\begin{array}{lll}
L_{\mathrm{g}} i_{\mathrm{gd}} & L_{\mathrm{g}} i_{\mathrm{gq}} & \frac{2}{3} C u_{\mathrm{dc}}
\end{array}\right]^{\mathrm{T}}, \quad H_{(\mathrm{b})}=\frac{1}{2} L_{\mathrm{g}} i_{\mathrm{gd}}^{2}+\frac{1}{2} L_{\mathrm{g}} i_{\mathrm{gq}}^{2}+\frac{1}{2}\left(\frac{2}{3} C\right) u_{\mathrm{dc}}^{2} \\
u_{(\mathrm{b})}=\left[\begin{array}{lll}
u_{\mathrm{gd}} & u_{\mathrm{gq}} & \frac{2 E_{\mathrm{g}}}{3 R_{\mathrm{b}}}
\end{array}\right]^{\mathrm{T}}, y_{(\mathrm{b})}=\left[\begin{array}{lll}
i_{\mathrm{gd}} & i_{\mathrm{gq}} & u_{\mathrm{dc}}
\end{array}\right]^{\mathrm{T}}
\end{array}\right. \\
& J_{(\mathrm{m})}=\left[\begin{array}{cccc}
0 & \omega L_{\mathrm{gm}} & 0 & 0 \\
-\omega L_{\mathrm{gm}} & 0 & 0 & 0 \\
0 & 0 & 0 & -S_{\mathrm{ch}} \\
0 & 0 & s_{\mathrm{ch}} & 0
\end{array}\right], R_{(\mathrm{m})}=\left[\begin{array}{cccc}
R_{\mathrm{gm}} & 0 & 0 & 0 \\
0 & R_{\mathrm{gm}} & 0 & 0 \\
0 & 0 & 0 & 0 \\
0 & 0 & 0 & 0
\end{array}\right], \\
& g_{(\mathrm{m})}=\left[\begin{array}{cccccc}
-u_{\mathrm{dcm}} & 0 & 1 & 0 & 0 & 0 \\
0 & -u_{\mathrm{dcm}} & 0 & 1 & 0 & 0 \\
0 & 0 & 0 & 0 & 1 & 0 \\
0 & 0 & 0 & 0 & 0 & 0
\end{array}\right]
\end{aligned}
$$

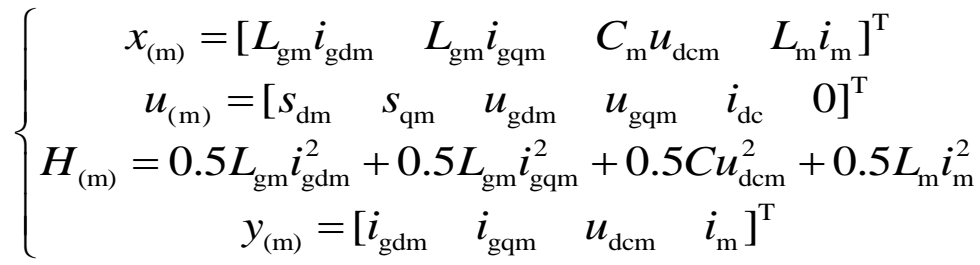

The matrices with the subscript (b) are for the BESS, and those with the subscript (m) are for the SMES. The essence of the IDA methodology is to configure the closed-loop control system as another PCH system as below:

$$
\begin{aligned}
& \dot{x}=\left[J_{\mathrm{d}}(x)-R_{\mathrm{d}}(x)\right] \nabla H_{\mathrm{d}}(x) \\
& \left\{\begin{array}{l}
J_{\mathrm{d}}(x)=J(x)+J_{\mathrm{a}}(x)=-J_{\mathrm{d}}^{\mathrm{T}}(x) \\
R_{\mathrm{d}}(x)=R(x)+R_{\mathrm{a}}(x)=R_{\mathrm{d}}^{\mathrm{T}}(x)>0 \\
H_{\mathrm{d}}(x)=H(x)+H_{\mathrm{a}}(x)
\end{array}\right.
\end{aligned}
$$

Where the matrices with the subscript $d$ are for the desired closed-loop HESS, and the matrices with the subscript a are those awaiting to be determined and can be interpreted as the parts brought by the closed-loop regulation. When the constraint requirements of the related matrices showed in (10) are meet, the system can achieve the globally asymptotic stability based on the Lyapunov stability theory. Considering that the balance positions of the closed-loop system should have the minimum energy, the desired energy functions which should be adapt to the closed-loop PCH configuration based on IDA methodology are determined as: 


$$
\begin{aligned}
H_{\mathrm{d}(\mathrm{b})} & =0.5 L_{\mathrm{g}}\left(i_{\mathrm{gd}}-i_{\mathrm{gd}}^{*}\right)^{2}+0.5 L_{\mathrm{g}}\left(i_{\mathrm{gq}}-i_{\mathrm{gq}}^{*}\right)^{2}+0.5(2 C / 3)\left(u_{\mathrm{dc}}-u_{\mathrm{dc}}^{*}\right)^{2} \\
H_{\mathrm{d}(\mathrm{m})}= & 0.5 L_{\mathrm{gm}}\left(i_{\mathrm{gdm}}-i_{\mathrm{gdm}}^{*}\right)^{2}+0.5 L_{\mathrm{gm}}\left(i_{\mathrm{gqm}}-i_{\mathrm{gqm}}^{*}\right)^{2} \\
& +0.5 C_{\mathrm{m}}\left(u_{\mathrm{dcm}}-u_{\mathrm{dcm}}^{*}\right)^{2}+0.5 L_{\mathrm{m}}\left(i_{\mathrm{m}}-i_{\mathrm{m}}^{*}\right)^{2}
\end{aligned}
$$

The core equation for designing PB control based on IDA methodology, which can be deduced by (5) and (10), is provided as below:

$$
\left(J_{\mathrm{d}}-R_{\mathrm{d}}\right)\left(-\nabla H_{\mathrm{a}}\right)=\left(J_{\mathrm{a}}-R_{\mathrm{a}}\right) \nabla H-g u
$$

IDA methodology can achieve the control law easily by choosing the underdetermined matrices and solving a group of simultaneous equations which can be obtained by (12). The underdetermined matrices in PCH models for BESS and SMES are chosen as follows:

$$
\begin{aligned}
& J_{\mathrm{a}(\mathrm{b})}=\left[\begin{array}{ccc}
0 & 0 & A_{1} \\
0 & 0 & A_{2} \\
-A_{1} & -A_{2} & 0
\end{array}\right], R_{\mathrm{a}(\mathrm{b})}=\left[\begin{array}{ccc}
R_{1} & 0 & 0 \\
0 & R_{1} & 0 \\
0 & 0 & R_{2}
\end{array}\right] \\
& \left\{\begin{array}{c}
A_{1}=-R_{1} i_{\mathrm{gd}}^{*} / u_{\mathrm{dc}}^{*}, A_{2}=-R_{1} i_{\mathrm{gq}}^{*} / u_{\mathrm{dc}}^{*} \\
R_{1}=2 u_{\mathrm{dc}}^{* 2} / 3 R_{\mathrm{b}}\left(i_{\mathrm{gd}}^{* 2}+i_{\mathrm{gq}}^{* 2}\right), R_{2}=-2 / 3 R_{\mathrm{b}}
\end{array}\right. \\
& J_{\mathrm{a}(\mathrm{m})}=\left[\begin{array}{cccc}
0 & 0 & 0 & 0 \\
0 & 0 & 0 & 0 \\
0 & 0 & 0 & 0 \\
0 & 0 & 0 & 0
\end{array}\right], R_{\mathrm{a}(\mathrm{m})}=\left[\begin{array}{cccc}
r & 0 & 0 & 0 \\
0 & r & 0 & 0 \\
0 & 0 & r_{1} & 0 \\
0 & 0 & 0 & r_{2}
\end{array}\right]
\end{aligned}
$$

By solving (12) and combining the integrator to improve the static properties which is proposed in ref [18], the final control laws of PB method are obtained as follows:

$$
\begin{aligned}
& s_{\mathrm{d}}=\left[-R_{\mathrm{g}} i_{\mathrm{gd}}^{*}+\omega L_{\mathrm{g}} i_{\mathrm{gq}}^{*}+u_{\mathrm{gd}}+R_{1}\left(i_{\mathrm{gd}}-i_{\mathrm{gd}}^{*}\right)-A_{1}\left(u_{\mathrm{dc}}-u_{\mathrm{dc}}^{*}\right)\right] / u_{d c}^{*} \\
& +K_{\mathrm{I}} \int u_{\mathrm{dc}}\left(i_{\mathrm{gd}}-i_{\mathrm{gd}}^{*}\right) d t-K_{\mathrm{I}} \int i_{\mathrm{gd}}\left(u_{\mathrm{dc}}-u_{\mathrm{dc}}^{*}\right) d t \\
& s_{\mathrm{q}}=\left[-R_{\mathrm{g}} i_{\mathrm{gq}}^{*}-\omega L_{\mathrm{g}} i_{\mathrm{gd}}^{*}+u_{\mathrm{gq}}+R_{1}\left(i_{\mathrm{gq}}-i_{\mathrm{gq}}^{*}\right)-A_{2}\left(u_{\mathrm{dc}}-u_{\mathrm{dc}}^{*}\right)\right] / u_{d c}^{*} \\
& +K_{\mathrm{I}} \int u_{\mathrm{dc}}\left(i_{\mathrm{gq}}-i_{\mathrm{gq}}^{*}\right) d t-K_{\mathrm{I}} \int i_{\mathrm{gq}}\left(u_{\mathrm{dc}}-u_{\mathrm{dc}}^{*}\right) d t \\
& s_{\mathrm{dm}}=\left[-R_{\mathrm{gm}} i_{\mathrm{gdm}}^{*}+r\left(i_{\mathrm{gdm}}-i_{\mathrm{gdm}}^{*}\right)+\omega L_{\mathrm{gm}} i_{\mathrm{gqm}}^{*}+u_{\mathrm{gdm}}\right] / u_{\mathrm{dcm}} \\
& +K_{\mathrm{Im} 1} \int u_{\mathrm{dcm}}\left(i_{\mathrm{gdm}}-i_{\mathrm{gdm}}^{*}\right) d t \\
& s_{\mathrm{qm}}=\left[-R_{\mathrm{gm}} i_{\mathrm{gqm}}^{*}+r\left(i_{\mathrm{gqm}}-i_{\mathrm{gqm}}^{*}\right)-\omega L_{\mathrm{gm}} i_{\mathrm{gdm}}^{*}+u_{\mathrm{gqm}}\right] / u_{\mathrm{dcm}} \\
& +K_{\mathrm{Im} 1} \int u_{\mathrm{dcm}}\left(i_{\mathrm{gqm}}-i_{\mathrm{gqm}}^{*}\right) d t \\
& s_{\mathrm{ch}}=-\left[r_{2} i_{\mathrm{m}}+\sqrt{r_{2}^{2} i_{\mathrm{m}}^{2}+4 r_{2} u_{\mathrm{dcm}}^{*}\left(r_{1} u_{\mathrm{dcm}}-r_{1} u_{\mathrm{dcm}}^{*}+i_{\mathrm{dc}}\right)}\right] / 2 u_{\mathrm{dcm}}^{*} \\
& +K_{\mathrm{Im} 2} \int i_{\mathrm{m}}\left(u_{\mathrm{dcm}}-u_{\mathrm{dcm}}^{*}\right) d t
\end{aligned}
$$


Where $K_{\mathrm{I}}$ is the integral coefficient for the BESS, $K_{\mathrm{Im} 1}$ and $K_{\operatorname{Im} 2}$ are the integral coefficients for the SMES.

\section{The HILS System for the Test MG}

A typical AC MG configuration as shown in Figure 3 for integrating the WG is considered to test the proposed HESS control method in this study. The diesel generator (DG) is critical for the normal operation of $\mathrm{MG}$ due to the relative unreliability of WG. For the sake of simplicity, the grid-connected mode of MG with the constant resistive load is considered for the test, but the proposed HESS is also applicable to other cases. In this study, HESS consisted of the BESS and SMES is employed to smooth the output power fluctuation of WG to improve the stability of the power system. The power of HESS $P_{\mathrm{H}}$ with bidirectional features adopts the charging as the positive direction. The storage capacity of HESS and the power rating of the other components in MG are provided in Figure 3. The initial state of charge (SOC) of the battery is $55 \%$, and the initial $i_{\mathrm{m}}$ is $350 \mathrm{~A}$.

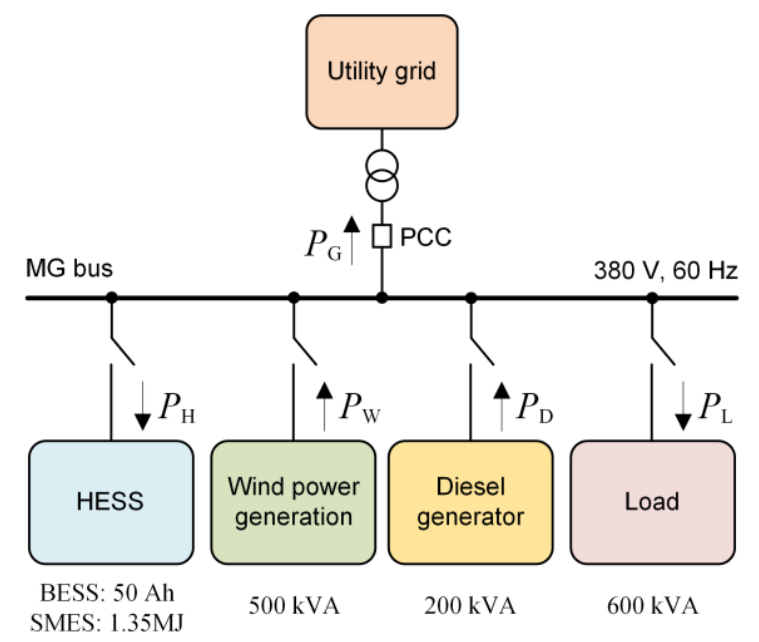

Figure 3. The Test MG System

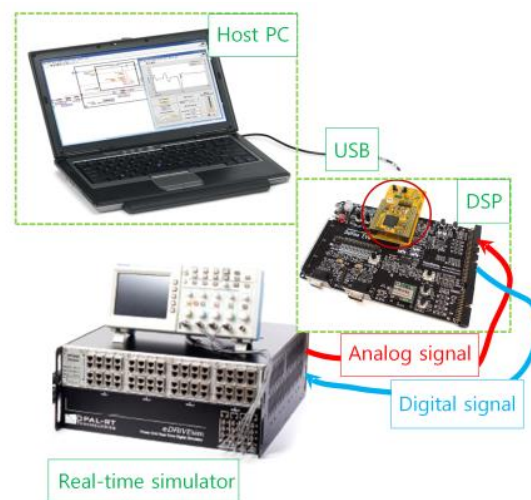

The principle of HILS system

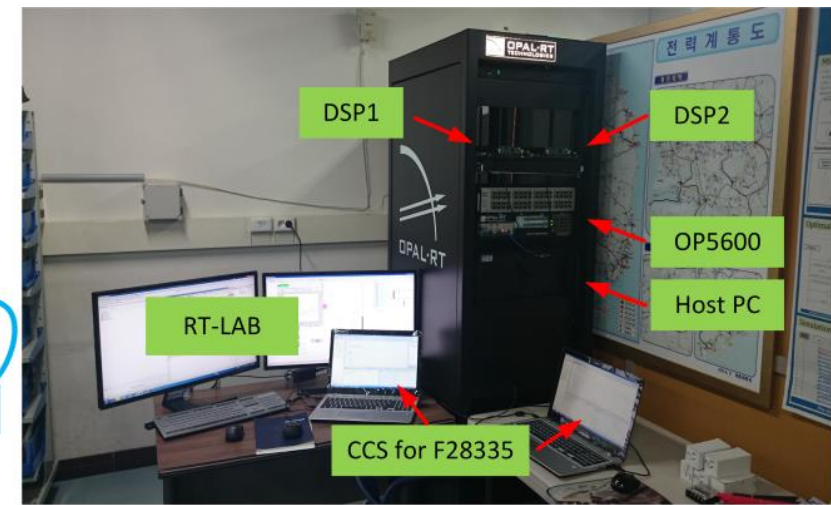

The HILS system configuration for the HESS test

Figure 4. The HILS System to Test the HESS Performance

The HESS in MG applications, a very complex system which was difficult to construct the experiment platform, can be tested by the HILS system conveniently with the aid of the real-time simulation approach. The principle of HILS system is 
illustrated in Figure 4. The real-time simulator which can simulate the hardware system by programming has the abundant analog and digital interface connected to the DSP controller. The analog outputs of the real-time simulator, which are usually the voltage, current, and power of the simulated test system, are sampled by the AD part of DSP to calculate the control output. Meanwhile, the digital outputs of DSP which are usually the PWM and switch signals are transferred to the real-time simulator to fulfill the closed-loop control. The real-time simulation can save a lot of time and expenses to build the hardware prototype and provide the credible results.

For the purpose of verifying the coordinated control of the HESS in MG, the OP5600 HILS system produced by OPAL-RT Technologies is employed in this study. Figure 4 shows the configuration of the HILS system for HESS test. The realtime simulator OP5600, which simulates the action of the test MG including the HESS, is programmed by the tool software RT-LAB in the host PC. The masterslave control structure is used to implement the coordinated control of the HESS. The host PC using RT-LAB software serves as the master controller which separates the power reference for BESS and SMES. Two DSPs (TMS320F28335) are used as the slave regulators to control the BESS and SMES respectively. Each DSP receives its power reference from the host PC and control the corresponding ESS by PB method.

\section{The Experimental Results based on HILS System}

In this section, the real-time simulation results for HESS test are presented. Table 1 provides the related parameters in the experiment.

Table 1. The Parameters in the Experiment

\begin{tabular}{|l|l|}
\hline The parameter name & The parameter value \\
\hline Sampling/switching frequency & $20 \mathrm{kHz} / 10 \mathrm{kHz}$ \\
\hline Battery nominal/fully-charged voltage & $800 \mathrm{~V} / 931.2 \mathrm{~V}$ \\
\hline $\boldsymbol{R}_{\mathrm{b}}, C_{,} L_{\mathrm{g},} \boldsymbol{R}_{\mathrm{g}} L_{\mathrm{g} 2}$ & $0.16 \Omega, 10000 \mu \mathrm{F}, 4 \mathrm{mH}, 1.1 \mathrm{~m} \Omega, 0.04 \mathrm{mH}$ \\
\hline$L_{\mathrm{m}}, C_{\mathrm{m}}, L_{\mathrm{gm},} \boldsymbol{R}_{\mathrm{gm},} L_{\mathrm{gm} 2}$ & $15 \mathrm{H}, 32000 \mu \mathrm{F}, 1.5 \mathrm{mH}, 1.1 \mathrm{~m} \Omega, 0.04 \mathrm{mH}$ \\
\hline $\mathrm{DC}$ voltage reference of SMIES & $800 \mathrm{~V}$ \\
\hline$C_{\mathrm{g}}, \boldsymbol{R}_{\mathrm{f}}, C_{\mathrm{fm}}, \boldsymbol{R}_{\mathrm{fm}}$ & $30 \mu \mathrm{F}, 0.1 \Omega, 30 \mu \mathrm{F}, 0.1 \Omega$ \\
\hline$r, r_{1}, \boldsymbol{r}_{2}$ & $1500,300,3000$ \\
\hline$k_{\mathrm{I}}, k_{\mathrm{Im} 1}, k_{\mathrm{Im} 2}$ & $0.2,3,0.35$ \\
\hline
\end{tabular}

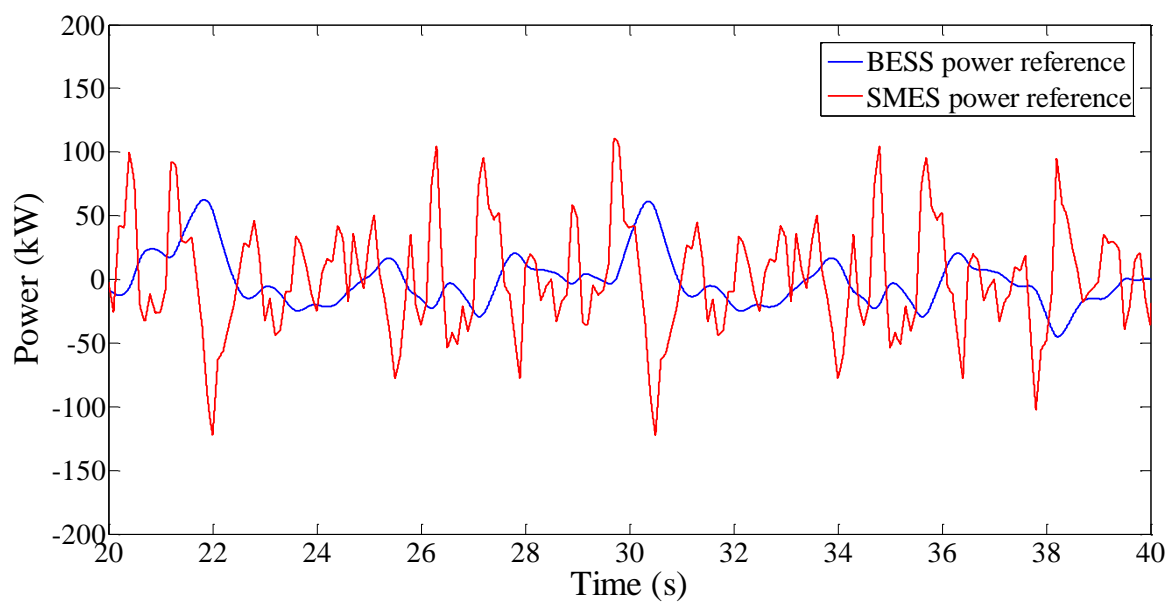

Figure 5. The Performance of Power Reference Division 
In the real-time simulation, the WG output power is generated by a group of wind speed data to simulate the fluctuation situations. Figure 5 provides the power reference division performance of HESS implemented by the master controller. It can be easily seen that the power reference of battery changes much more slowly in compared with that of SMES with the aid of LPF2, which might contribute to the protection of the battery service life. The power reference division performance which is consistent with the expectations proves the effectiveness of the proposed power division method.

Figure 6 and Figure 7 depict the power tracking performance of BESS and SMES respectively. In figure 6 , it can be found that BESS tracks the low-frequency reference power well with a very small switching ripple. Some slight BESS power fluctuation in the tracking process might be attributed to the large value of $L_{\mathrm{g}}$ (4 $\mathrm{mH})$. The power tracking performance and ripple levels have a compromise. Nevertheless, the overall performance of BESS considered the power tracking and ripple filtering is satisfactory. In contrast with BESS, SMES should have a much better power tracking ability because of the high-frequency reference. Hence a smaller connection inductance $(1.5 \mathrm{mH})$ is adopted in SMES compared to BESS. It can be observed from Figure 7 that SMES tracks the high-frequency power reference well with an acceptable ripple degree.

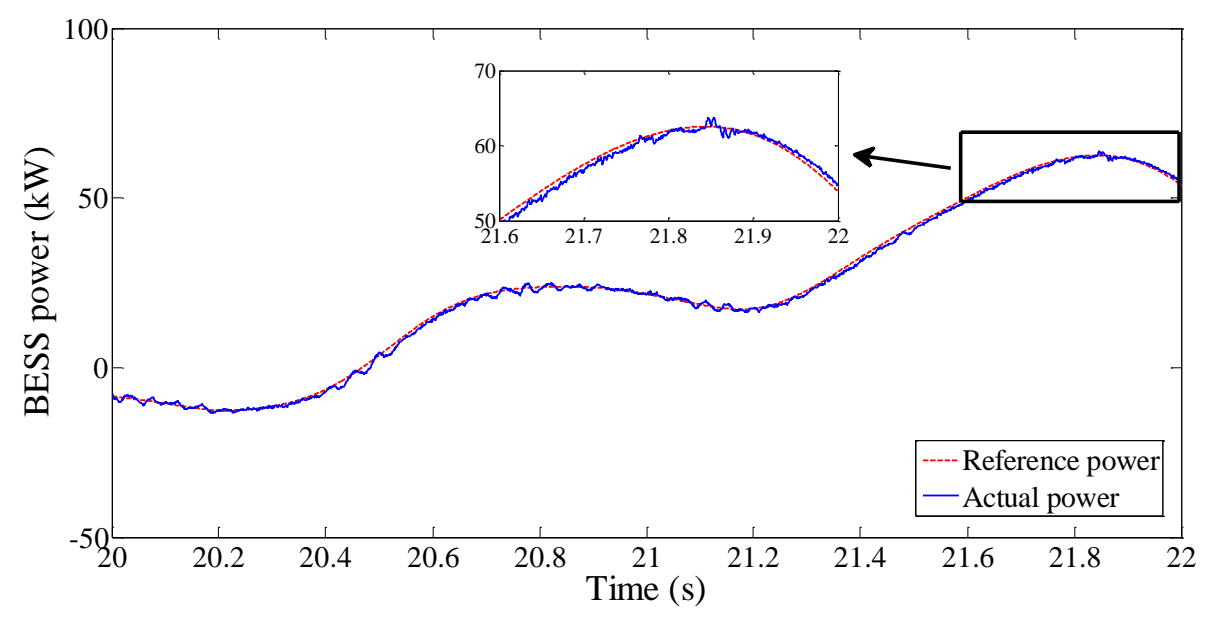

Figure 6. The Performance of BESS Power Tracking

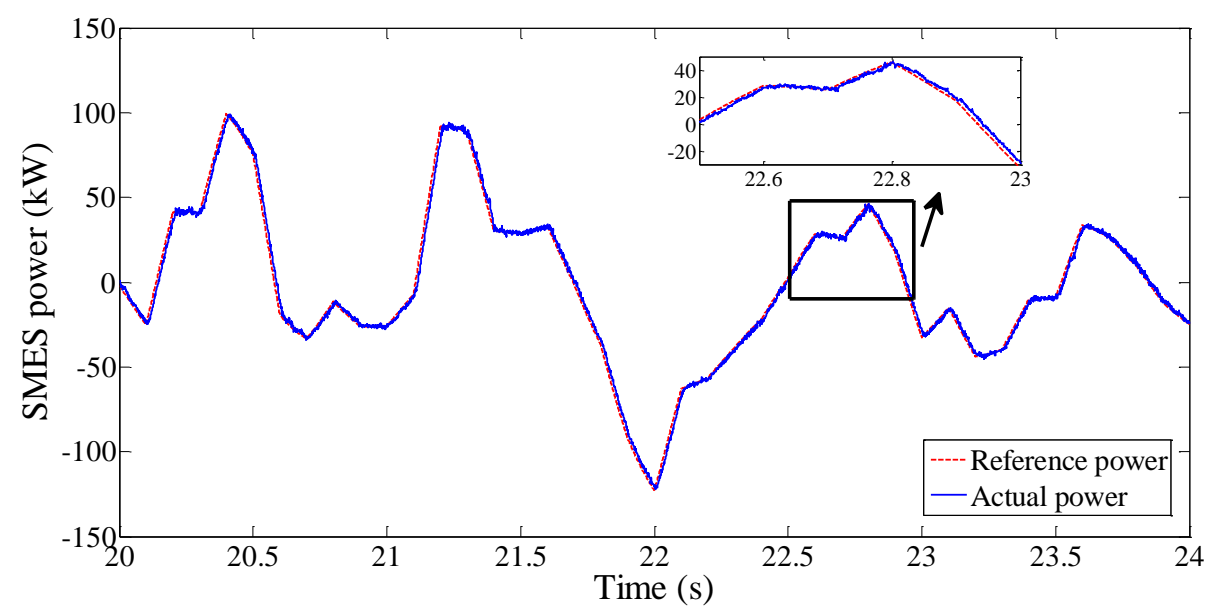

Figure 7. The Performance of SMES Power Tracking 
Figure 8 focuses on the dynamic performance of DC voltage in SMES when HESS is put into operation. It can be seen that the DC voltage of SMES reaches to the reference value very fast $(0.02 \mathrm{~s})$ without any overshoot during the regulation process. The outstanding dynamic performance proves the good transient control performance of proposed PB controller. Also, the ripple of DC voltage is very small as is showed in the enlarged figure. Nonetheless, there is a very slight steady error caused by the inevitable $\mathrm{AD}$ offset.

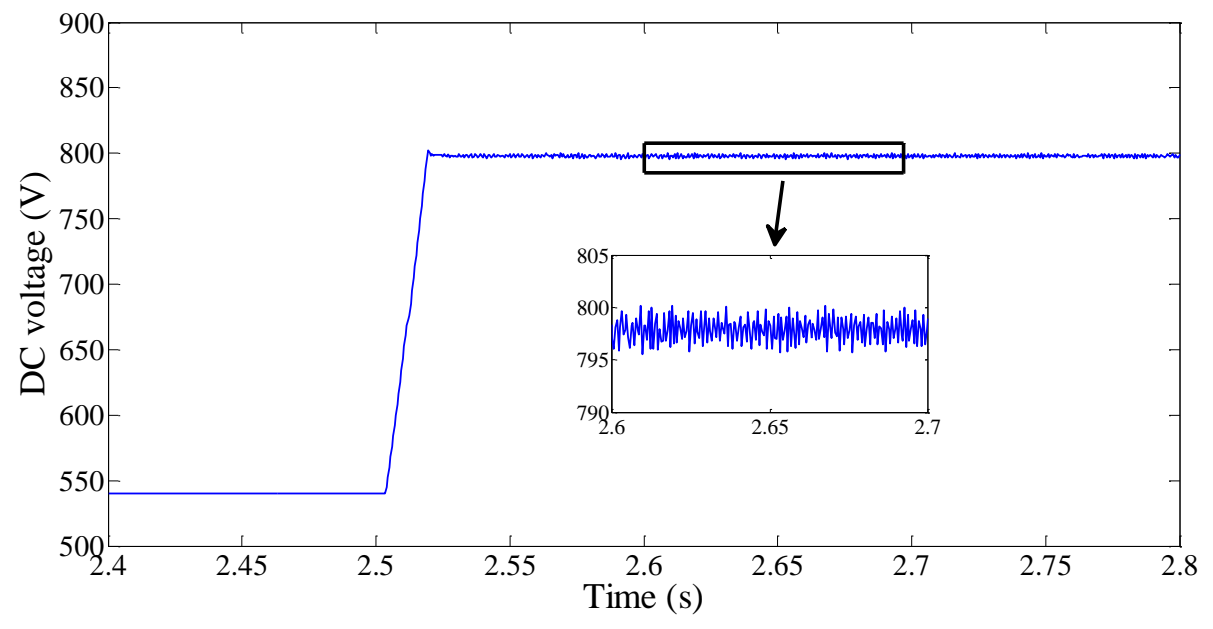

Figure 8. The Dynamic Performance of DC Voltage in SMES

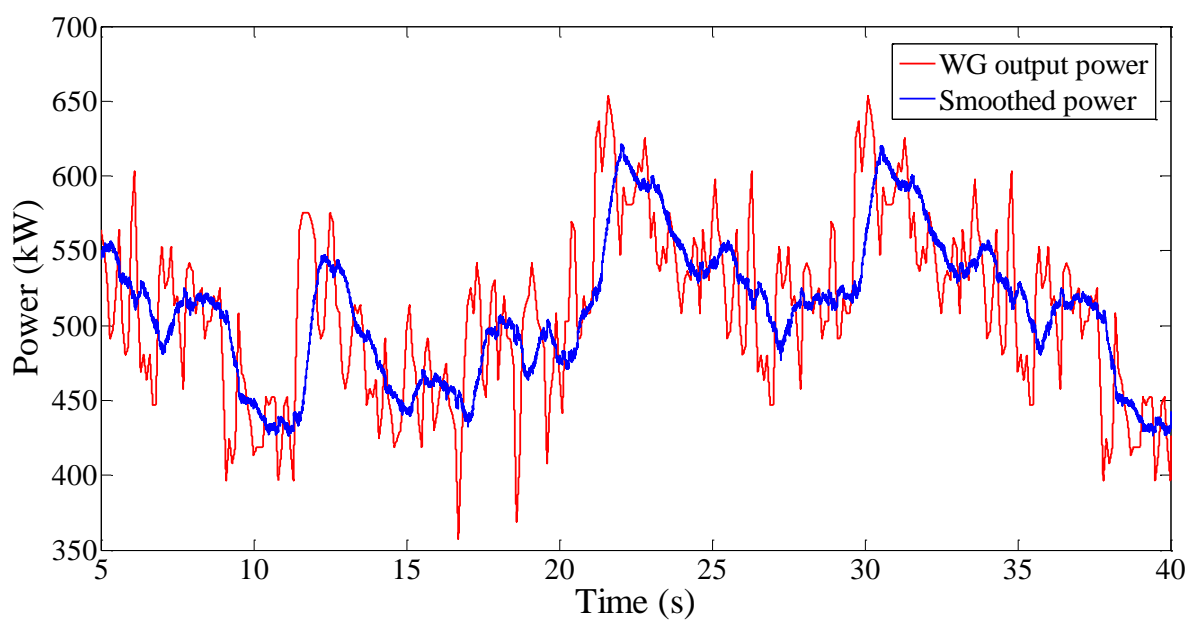

Figure 9. The Performance of Smoothing WG Power by HESS

Figure 9 demonstrates the overall performance of HESS for smoothing the fluctuation of WG output power. The smoothed power tracks the changing trend of WG power with the aid of LPF1. Furthermore, most of the fluctuation components are absorbed by the HESS, and the smoothed WG power varies slowly with a small switching ripples. HESS which is used as a power buffer to improve the power system stability has a satisfactory overall performance with the proposed coordination control method.

\section{Conclusions}

In this study, a coordinated control strategy of HESS used to smooth the WG power fluctuations has been proposed. One second-order LPF with a very low cutoff frequency was employed to track the variation trend of WG power and determine the total power 
reference of HESS. Another second-order LPF which was served as the power reference separator for HESS could optimize the charge-discharge current for battery and extend its service life. The nonlinear PB current regulator was used to control HESS due to its good control performance. The detailed design process of PB current control for HESS was presented in this paper.

For the purpose of verifying the proposed coordination control of HESS, the real-time simulation by the HILS system has been implemented using the master-slave control approach. The simulation results showed that the power reference of the battery changed slowly, which might contribute to the battery protection. It could also be found in the simulation that HESS tracked the reference power very well both in transient and steady states. Furthermore, the smoothed WG power by HESS was much flatter than the original WG power, which would improve the stability of the power grid. These experimental results have proved the correctness and validity of the proposed coordination control strategy for HESS.

\section{Acknowledgments}

This work was supported under the framework of international cooperation program managed by the National Research Foundation of Korea (NRF-2015K2A2A2002148).

\section{References}

[1] E. Mayhorn, L. Xie, and K. Butler-Purry, "Multi-time Scale Coordination of Distributed Energy Resources in Isolated Power Systems", IEEE Transactions on Smart Grid, vol. 8, no.2, (2017), pp. 9981005.

[2] T. Ma, M. H. Cintuglu, and O. Mohammed, "Control of a Hybrid AC/DC Microgrid Involving Energy Storage and Pulsed Loads", IEEE Transactions on Industry Applications, vol. 53, no. 1, (2017), pp. 567575.

[3] D. B. W. Abeywardana, B. Hredzak, and V. G. Agelidis, "A Fixed-Frequency Sliding Mode Controller for a Boost-Inverter-Based Battery-Supercapacitor Hybrid Energy Storage System”, IEEE Transactions on Power Electronics, vol.32, no. 1, (2017), pp. 668-680.

[4] J. Shen, and A. Khaligh, "Design and real-time controller implementation for a battery-ultracapacitor hybrid energy storage system", IEEE Transactions on Industrial Informatics, vol. 12, no. 5, (2016), pp. 1910-1918.

[5] D. B. W. Abeywardana, B. Hredzak, V. G. Agelidis, and G. D. Demetriades, "Supercapacitor Sizing Method for Energy-Controlled Filter-Based Hybrid Energy Storage Systems", IEEE Transactions on Power Electronics, vol. 32, no. 2, (2017), pp. 1626-1637.

[6] Z. X. Zheng, X. Y. Xiao, C. S. Li, Z. Chen, and Y. Zhang, "Performance Evaluation of SMES System for Initial and Steady Voltage Sag Compensations", IEEE Transactions on Applied Superconductivity, vol. 26, no. 7, (2016), 5701105.

[7] S. Hu, Z. Liang, D. Fan, and X. He, "Hybrid Ultracapacitor-Battery Energy Storage System Based on Quasi-Z-source Topology and Enhanced Frequency Dividing Coordinated Control for EV", IEEE Transactions on Power Electronics, vol. 31, no. 11, (2016), pp. 7598-7610.

[8] N. Mendis, K. M. Muttaqi, and S. Perera, "Management of Low- and High-Frequency Power Components in Demand-Generation Fluctuations of a DFIG-Based Wind-Dominated RAPS System Using Hybrid Energy Storage”, IEEE Transactions on Industry Applications, vol. 50, no. 3, (2014), pp. 2258-2268.

[9] N. R. Tummuru, M. K. Mishra, and S. Srinivas, "Dynamic energy management of hybrid energy storage system with high-gain PV converter”, IEEE Transactions on Energy Conversion, vol. 30, no. 1, (2015), pp. 150-160.

[10] H. Mahmood, D. Michaelson, and J. Jiang, "A Power Management Strategy for PV/Battery Hybrid Systems in Islanded Microgrids", IEEE Journal of Emerging and Selected Topics in Power Electronics, vol. 2, no. 4, (2014), pp. 870-882.

[11] J. Shen, and A. Khaligh, "Design and real-time controller implementation for a battery-ultracapacitor hybrid energy storage system”, IEEE Transactions on Industrial Informatics, vol. 12, no. 5, (2016), pp. 1910-1918.

[12] Q. Jiang, and H. Hong, "Wavelet-Based Capacity Configuration and Coordinated Control of Hybrid Energy Storage System for Smoothing Out Wind Power Fluctuations", IEEE Trans. on Power Systems, vol. 28, no. 2, (2013), pp. 1363-1372. 
[13] J. Zeng, Z. Zhang, and W. Qiao, "An Interconnection and Damping Assignment Passivity-Based Controller for a DC-DC Boost Converter With a Constant Power Load", IEEE Trans. Industry Applications, vol. 50, no. 4, (2014), pp. 2314-2322

[14] K. Nunna, M. Sassano, and A. Astolfi, "Constructive interconnection and damping assignment for portcontrolled Hamiltonian systems", IEEE Transactions on Automatic Control, vol. 60, no. 9, (2015), pp. 2350-2361.

[15] R. Hou, T. T. Nguyen, H. M. Kim, H. Song, and Y. Qu, "An Energy-Based Control Strategy for Battery Energy Storage Systems: A Case Study on Microgrid Applications”, Energies, vol. 10, no. 2, (2017), 215.

[16] S. I. Seleme, S. R. Silva, and L. T. L. Soares, "Stabilization of Back-to-Back converter in wind generation system connected to the grid: IDA-PBC versus PI Control", Proceedings of 2014 16th European Conference on Power Electronics and Applications (EPE'14-ECCE Europe), Lappeenranta, Finland, (2014) August 26-28.

[17] O. Tremblay, L. A. Dessaint, and A. I. Dekkiche, "A Generic Battery Model for the Dynamic Simulation of Hybrid Electric Vehicles", Proceedings of the 2007 IEEE Vehicle Power and Propulsion Conference (VPPC), Texas, USA, (2007) September 9-12, pp. 284-289.

[18] A. Donaire, and S. Junco, "On the addition of integral action to port-controlled Hamiltonian systems", Automatica, vol. 45, no. 8, (2009), pp. 1910-1916. 